\title{
Aplicação de protocolo de aceleração da recuperação pós-operatória em cirurgias de colectomia retossigmoidectomia, pancreatectomia e hepatectomia: experiência de hospital terciário
}

\author{
Application of postoperative recovery acceleration protocol in rectosigmoidectomy, pancrea- \\ tectomy and hepatectomy colectomy surgeries: experience of a tertiary hospital
}

\section{DOI: 10.37111/braspenj.2019344006}

\author{
Fernanda de Matos Feijó' \\ Marcelo Campos Appel-da-Silva ${ }^{2}$ \\ Marcio Fernando Boff 3 \\ Caroline Schardong Boligon' \\ Mara Regina Santos da Silva' \\ Daniê Weber \\ Cristine Nascente Igansi ${ }^{4}$
}

\begin{abstract}
Unitermos:
Terapia nutricional. Assistência perioperatória. Cuidados pós-operatórios. Complicações pós-operatórias. Protocolos clínicos. Guia de prática clínica.
\end{abstract}

\section{Keywords:}

Nutrition therapy. Perioperative care. Postoperative care. Postoperative complications. Clinical protocols. Practice guideline.

\section{Endereço para correspondência: \\ Caroline Schardong Boligon \\ Hospital Mãe de Deus}

Rua José de Alencar, 286 - Porto Alegre, RS.

E-mail: caroboligon@gmail.com

\section{Submissão}

14 de junho de 2019

\section{Aceito para publicação}

16 de agosto de 2019

\begin{abstract}
RESUMO
Introdução: A recuperação pós-operatória dos pacientes submetidos a procedimentos cirúrgicos tem sido foco de atenção nos últimos anos. Baseado nos resultados favoráveis do protocolo ERAS (Enhanced Recovery After Surgery, sigla em inglês), foi desenhado no Brasil o protocolo ACERTO (Aceleração da Recuperação Total pós-operatória). Este protocolo define algumas rotinas perioperatórias, como terapia nutricional, diminuição do período de jejum pré-operatório, realimentação precoce no pós-operatório, diminuição da hidratação venosa, entre outros, com impacto favorável na recuperação pós-operatória. $O$ objetivo deste estudo foi comparar a resposta de pacientes submetidos a procedimentos cirúrgicos seguindo ou não um protocolo piloto de aceleração da recuperação pós-operatória. Método: Estudo de caso controle aninhado a um estudo transversal, em que foram revisados prontuários de pacientes que internaram para realização de procedimentos de colectomia, retossigmoidectomia, pancreatectomia e hepatectomia, entre 2016 e 2017. Os pacientes foram divididos em grupo intervenção e controle. Os principais desfechos analisados foram: tempo de internação, desfecho, taxa de reinternação e custo hospitalar. Resultados: Foram coletados dados de 76 pacientes ( 30 no grupo intervenção e 46 no grupo controle). A mediana de tempo de internação foi de 6,5 dias no grupo intervenção e 13,5 dias no grupo controle $(p=0,0001)$. A taxa de reinternação em 30 dias após alta foi 5,3 vezes menor no grupo intervenção quando comparado ao grupo controle $(p=0,02)$. Em relação aos custos, - grupo intervençẫo apresentou uma mediana de custo de R\$15.493,91 e o grupo controle, R\$25.929,19 ( $p=0,0036$ ). Conclusão: Os pacientes que receberam a intervenção do protocolo multimodal de aceleração da recuperação pós-operatória apresentaram menor tempo de internação, menor taxa de reinternação em 30 dias e menor custo hospitalar quando comparados aos pacientes do grupo controle.
\end{abstract}

\section{ABSTRACT}

Introduction: Postoperative recovery of patients undergoing surgical procedures has been a focus of attention in recent years. Positive results from the Enhanced Recovery Based on the favorable results of the Enhanced Recovery After Surgery (ERAS) protocol, the ACERTO (Aceleração da Recuperação Total pós-operatória) protocol was designed in Brazil. This protocol defines some perioperative routines, such as nutritional therapy, decreased preoperative fasting period, early postoperative feeding, decreased venous hydration, among others, with favorable impact on postoperative recovery. The aim of this study was to compare the response of patients undergoing surgical procedures following or not a pilot protocol for accelerating postoperative recovery. Methods: A case-control study nested in a cross-sectional study reviewing medical records of patients hospitalized for colectomy, rectosigmoidectomy, pancreatectomy, and hepatectomy procedures between 2016 and 2017. The patients were divided into intervention and control group. Main endpoints analyzed were: length of postoperative hospital stay, hospitalization outcomes, rate of readmission within 30 days, and hospital costs. Results: 76 patients were enrolled (30 in intervention group and 46 in control group). The median length of in-hospital stay was 6.5 days for the intervention group and 13.5 days for the control group $(p=0.0001)$. Rate of readmission within 30 days was 5.3 times lower in the intervention group in comparison to control group $(p=0.02)$. Cost analysis showed a median per-patient cost of $R \$ 15.493,91$ in the intervention group compared to a median per-patient cost of $R \$ 25.929,19$ in the control group $(p=0.0036)$. Conclusion: Patients who underwent the intervention of the postoperative recovery acceleration multimodal protocol presented shorter hospital stay, lower readmission rate at 30 days and lower hospital costs when compared to patients in the control group.

1. Nutricionista, Serviço de Nutrição Clínica/ Equipe Multiprofissional de Terapia Nutricional, Hospital Mãe de Deus, Porto Alegre, RS, Brasil.

2. Médico Gastroenterologista, Serviço de Gastroenterologia e Endoscopia e Equipe Multidisciplinar de Terapia Nutricional (EMTN), Hospital Mãe de Deus, Porto Alegre, RS, Brasil.

3. Cirurgião Oncológico, Hospital do Câncer/ Hospital Mãe de Deus, Porto Alegre, RS, Brasil.

4. Epidemiologista, Serviço de Qualidade, Segurança do Paciente e Controle de Infecção, Hospital Mãe de Deus, Porto Alegre, RS, Brasil. 


\section{INTRODUÇ̃̃O}

A recuperação pós-operatória dos pacientes submetidos a procedimentos cirúrgicos tem sido foco de atenção nos últimos anos, tendo por base o novo paradigma da medicina baseada em evidências, novos protocolos de cuidados ao paciente cirúrgico começaram a surgirl-3.

O protocolo do grupo ERAS (Enhanced Recovery After Surgery, sigla em inglês), formado por médicos, especialmente por cirurgiões colorretais, em 2001, e realizado na Europa, foi publicado na forma de consenso'. Este protocolo tem por base estudos controlados, randomizados e metanálises, que demonstraram que a utilização do protocolo apoiada pela prática da medicina baseada em evidências quando aplicados à cirurgia abdominal, podem prover um retorno precoce da função intestinal e melhoria das funções fisiológicas dos pacientes ${ }^{4}$.

Em 2005, baseado no protocolo ERAS, foi desenhado no Brasil, o protocolo ACERTO (Aceleração da Recuperação Total pós-operatória), com base em uma grande revisão bibliográfica sobre cuidados perioperatórios, iniciou um programa multimodal pioneiro ${ }^{4}$. O protocolo ACERTO define algumas rotinas de prescrição perioperatória, como terapia nutricional, diminuição do período de jejum préoperatório, realimentação precoce no pós-operatório, diminuição da hidratação venosa, entre outros, com impacto favorável na recuperação pós-operatória, redução do tempo de internação e de custos hospitalares ${ }^{4,5}$. $\bigcirc$ objetivo do presente trabalho foi comparar a resposta de pacientes submetidos a procedimentos cirúrgicos, seguindo ou não um protocolo piloto de aceleração da recuperação pós-operatória.

\section{MÉTODO}

\section{Delineamento do Estudo}

Foi realizado um estudo de caso controle aninhado a um estudo transversal de revisão de prontuários, entre os anos de 2016 e 2017, de pacientes que submetidos a procedimentos cirúrgicos pelo Serviço de Cirurgia do Hospital Mãe de Deus, localizado na Zona Sul de Porto Alegre.

\section{População e Amostra}

A população do estudo é originária de pacientes que internaram para realização de procedimentos cirúrgicos pelo Serviço de Cirurgia do Hospital Mãe de Deus.

A amostra do estudo consiste de 76 pacientes que realizaram procedimentos cirúrgicos de colectomia, retossigmoidectomia, pancreatectomia e hepatectomia no período. Para participar do estudo, os pacientes teriam que preencher os critérios de elegibilidade, ou seja, ser maior de 18 anos e ter realizado o procedimento no Hospital Mãe de Deus.
Grupo Intervenção $(n=30)$ : foram considerados casos todos os pacientes que realizaram procedimentos cirúrgico de colectomia e/ou retossigmoidectomia, pancreatectomia, hepatectomia e submetidos ao Protocolo ACERTO ${ }^{4}$, com abordagem multidisciplinar focada em atendimento perioperatório especializado.

Grupo Controle $(n=46)$ : foram considerados potenciais controles os pacientes que realizaram procedimentos cirúrgicos de colectomia retossigmoidectomia, hepatecomia e pancreatectomia e não foram submetidos ao Protocolo ACERTO ${ }^{4}$.

Todos os pacientes elegíveis nesse estudo, tanto para o grupo intervenção como controle, preencheram os critérios acima, considerando uma proporção de 1,5 controles para cada caso.

Para ambos os grupos foram pesquisados os dados antropométricos, tempo de internação, desfecho, taxa de reinternação e custo hospitalar para cada paciente.

\section{Variáveis do Estudo}

Foram avaliados, em prontuário médico, as seguintes variáveis: aderência aos itens do protocolo de aceleração da recuperação pós-operatória; complicações transoperatórias; complicações pós-operatórias; tempo de internação em unidade de terapia intensiva (UTI); tempo total de internação; frequência de reinternação hospitalar dentro de 30 dias; custos hospitalares; desfecho.

\section{Análise dos Dados}

Os dados foram registrados e armazenados em um banco de dados, com acesso restrito aos pesquisadores. Para a análise estatística foi utilizado o programa SPSS ${ }^{\circledR}$ (Statistical Package for the Social Sciences) versão 20.0. As variáveis contínuas foram descritas como média e desvio padrão ou mediana e intervalo interquartil. As variáveis categóricas foram descritas como número e percentual. Para comparação de variáveis contínuas foi utilizado teste t de Student ou teste de Mann-Whitney. Para avaliar associação entre variáveis categóricas foi utilizado teste Qui-quadrado ou Exato de Fisher. Valor de $p<0,05$ foi considerado significativo para todas as análises. O odds ratio (OR) ou razão de chances (RC) foi calculado para as análises uni/bivariadas, por meio do método de regressão logística, com intervalo de confiança de $95 \%$.

O presente estudo foi aprovado pelo Comitê de Ética de Pesquisas com Seres Humanos do Hospital Mãe de Deus, estando de acordo com as normas vigentes na Resolução n 466/2012 do Conselho Nacional de Saúde/ Ministério da Saúde, que regulamentam a pesquisa envolvendo seres humanos. 


\section{RESULTADOS}

No período de 2016 a 2017, foram arrolados 76 pacientes, sendo 30 do grupo intervenção e 46 do grupo controle.

A mediana de idade dos pacientes foi de 67 (34-94) anos. Os pacientes do grupo intervenção apresentaram uma mediana de idade de 56,5 anos e do grupo controle, 71,5 anos. Houve diferença estatisticamente significativa quanto à idade ao se comparar os dois grupos, sendo o grupo intervenção mais jovem do que o grupo controle ( $p$ $\leq 0,006$ ). Sendo assim, temos que os casos são mais jovens que os controles, o que pode gerar um confundimento nas análises de desfechos, em especial ao que se refere ao tempo de internação/tempo de recuperação/custos, por se tratar de pacientes com idade inferior. Para minimizarmos esse efeito, realizamos o pareamento de casos e controles, ou seja, selecionamos controles de forma que eles fossem similares aos casos em certas características, como idade, sexo, comorbidades, ou variáveis que julgarmos ser variáveis de confundimento no estudo.

Quanto ao sexo, $44(57,9 \%)$ pacientes eram do sexo feminino, e $32(42,1 \%)$ do sexo masculino. Não foi observada diferença significativa entre os grupos intervenção e controle quanto ao sexo $(p=0,67)$ (Tabela 1).

Em relação ao tempo de internação, o grupo intervenção apresentou mediana de tempo de internação de 6,5 dias, e o grupo controle, de 13,5 dias. Observou-se diferença estatisticamente significativa entre os grupos intervenção e controle em relação ao tempo de internação $(p=0,0001)$. Sendo assim, o grupo intervenção apresentou tempo de internação menor em relação ao grupo controle.

Quanto ao desfecho reinternação em 30 dias após alta, o grupo intervenção apresentou 5,3 vezes menos chance de reinternar quando comparado ao grupo controle (Tabela 2).

Em relação aos custos, o grupo intervenção apresentou mediana de custo de $R \$ 15.493,91$ ( $R \$ 9.100$ - $R \$$ $42.424,54)$ e o grupo controle, $R \$ 25.929,19$ ( $R \$ 6.435,02$ - $R \$ 136.615,61)$. Observou-se diferença estatisticamente significativa entre os grupos em relação aos custos, sendo de 8,49 vezes menor no grupo intervenção em relação ao grupo controle $(p=0,0036)$.

Tabela 1 - Distribuição das características sociodemográficas de acordo com o grupo de estudo arrolado (grupo intervenção e grupo controle).

\begin{tabular}{lccc}
\hline Variáveis & $\begin{array}{c}\text { Grupo Intervenção } \\
(\mathbf{n}=30)\end{array}$ & $\begin{array}{c}\text { Grupo Controle } \\
(\mathbf{n}=46)\end{array}$ & Valor $\mathbf{P}^{*}$ \\
\hline Sexo & & & 0,67 \\
Masculino & $14(46,7 \%)$ & $18(39,1 \%)$ & \\
Feminino & $16(53,3 \%)$ & $28(60,9 \%)$ & \\
\hline
\end{tabular}

* Valor de p para testes t de Student ou Mann-Whitney.
Tabela 2 - Razão de chances ajustadas e IC 95\% da associação entre a variável reinternação em até 30 dias e utilização do protocolo ACERTO.

\begin{tabular}{lc}
\hline Variável & $\begin{array}{c}\text { Utilização do Protocolo } \\
\text { ACERTO }\end{array}$ \\
\hline Reinternação $\leq \mathbf{3 0}$ dias $\left(\right.$ IC95\%) ${ }^{*}$ \\
$\quad$ Sim \\
Não & $5,34(1,18-84,1)$ \\
\hline Razão de Chances (RC); Intervalo de Confiança para o RC de $95 \%($ IC95\%) \\
${ }^{*}$ RC ajustada para a variável da tabela
\end{tabular}

\section{DISCUSSÃO}

Nos últimos anos, diversos estudos demonstraram resultados positivos com a aplicação de programas multimodais, na recuperação pós-operatória de cirurgias de grande porte ${ }^{6-11}$.

Nossos dados demonstraram que os pacientes que receberam a intervenção do protocolo multimodal de aceleração da recuperação pós-operatória apresentaram menor tempo de internação. Esses dados corroboram com achados da literatura em que protocolos de recuperação multimodal resultaram em redução do tempo de internação em torno de $30 \%$ a $50 \%{ }^{7}$. Em estudo de Nelson et al. ${ }^{8}$, observou-se que houve redução significativa no tempo de permanência de um período pré-protocolo ERAS (média de 9,8 dias) versus (média de 7,5 dias) no pós-protocolo ERAS.

Em relação à reinternação em 30 dias pós alta, o grupo intervenção apresentou 5,3 vezes menos chances de reinternar em 30 dias quando comparado ao grupo controle. Esse achado corrobora com um estudo de metanálise de Varadhan et al. ${ }^{9}$, em que demonstraram que não houve aumento no percentual de reinternações motivado pelo emprego de protocolos multimodais. Nelson et al. ${ }^{8}$ observaram uma redução significativa no risco de readmissão, comparando pacientes pré protocolo ERAS com os pós ERAS. O risco de readmissão após a alta de 30 dias foi 1,73 vezes maior nos pacientes que não realizaram o protocolo multimodal.

$\bigcirc$ presente estudo identificou que o grupo intervenção apresentou custo significativamente menor quando comparado ao grupo controle, como também pode ser observado em um estudo retrospectivo em cirurgias da coluna vertebral desenvolvido por Wang e Grossman "1 , que observaram uma economia de $\$ 3442,00$ ou $15,2 \%$ por procedimento com a aplicação dos métodos ERAS. Nelson et al. ${ }^{8}$ examinaram o efeito da diretriz ERAS nos resultados dos pacientes em tempo de permanência, complicações e readmissões pós-alta, em um sistema de saúde. A análise econômica indicou que, após contabilizar custos de intervenção, reduções no tempo de permanência hospitalar, complicações e readmissões geraram uma economia líquida de custos entre 1.955 .758 a 4.110 .977 dólares ou 2,806 a 5,898 dólares por paciente ${ }^{8}$. 


\section{CONCLUSÃO}

No presente estudo, os pacientes que receberam a intervenção do protocolo multimodal de aceleração da recuperação pós-operatória apresentaram menor tempo de internação, menor taxa de reinternação em 30 dias e menor custo hospitalar quando comparados aos pacientes do grupo controle.

Esses resultados demonstram a importância da implementação de protocolos multimodais de aceleração da resposta pós-operatória na recuperação do paciente e na redução de custos hospitalares.

Trabalhos futuros incluem aumento da amostra e inclusão de novas variáveis.

\section{REFERÊNCIAS}

1. Nygren J, Thacker J, Carli F, Fearon KC, Norderval S, Lobo DN, et al; Enhanced Recovery After Surgery Society. Guidelines for perioperative care in elective rectal/pelvic surgery: Enhanced Recovery After Surgery (ERAS $\left.{ }^{\circledR}\right)$ Society recommendations. Clin Nutr. 2012;31(6):801-16.

2. Aguilar-Nascimento JE, Bicudo-Salomão A, Caporossi C, Silva RM, Cardoso EA, Santos TP. Acerto pós-operatório: avaliação dos resultados da implantação de um protocolo multidisciplinar de cuidados peri-operatórios em cirurgia geral. Rev Col Bras Cir. 2006;33(3):181-8.

3. Fearon KC, Ljungqvist O, Von Meyenfeldt M, Revhaug A, Dejong $\mathrm{CH}$, Lassen $\mathrm{K}$, et al. Enhanced recovery after surgery: a consensus review of clinical care for patients undergoing colonic resection. Clin Nutr. 2005;24(3):466-77.

4. Bicudo-Salomão A, Meireles MB, Caporossi C, Crotti PLR, Aguilar-Nascimento JE. Impact of the ACERTO project in the postoperative morbi-mortality in a university hospital. Rev Col Bras Cir. 2011;38(1):3-10.

5. Aguilar-Nascimento JE, Salomão AB, Caporossi C, Diniz BN. Clinical benefits after the implementation of a multimodal perioperative protocol in elderly patients. Arq Gastroenterol. 2010;47(2):178-83.

6. Aguilar-Nascimento JE, Salomão AB, Waitzberg DL, DockNascimento DB, Correa MITD, Campos ACL, et al. ACERTO guidelines of perioperative nutritional interventions in elective general surgery. Rev Col Bras Cir. 2017;44(6):633-48.

7. Ljungqvist $\mathrm{O}$, Scott M, Fearon KC. Enhanced recovery after surgery: a review. JAMA Surg. 2017;152(3):292-8.

8. Nelson G, Kiyang LN, Crumley ET, Chuck A, Nguyen T, Faris $\mathrm{P}$, et al. Implementation of Enhanced Recovery After Surgery (ERAS) across a provincial healthcare system: the ERAS Alberta colorectal surgery experience. World J Surg. 2016;40(5):1092-103.

9. Varadhan KK, Neal KR, Dejong CH, Fearon KC, Ljungqvist O, Lobo DN. The enhanced recovery after surgery (ERAS) pathway for patients undergoing major elective open colorectal surgery: a meta-analysis of randomized controlled trials. Clin Nutr. 2010;29(4):434-40.

10. Dietz N, Sharma M, Adams S, Alhourani A, Ugiliweneza B, Wang D, et al. Enhanced Recovery After Surgery (ERAS) for spine surgery: a systematic review. World Neurosurg. 2019;130:415-26.

11. Wang MY, Grossman J. Endoscopic minimally invasive transforaminal interbody fusion without general anesthesia: initial clinical experience with 1-year follow-up. Neurosurg Focus. 2016;40(2):E13.

Local de realização do estudo: Serviço de Cirurgia do Hospital Mãe de Deus, Porto Alegre, RS, Brasil.

Conflito de interesse: Os autores declaram não haver.

Foi apresentado como trabalho de destaque no XXIII Congresso Brasileiro de Nutrição Parenteral e Enteral, entre 20 a 23 de outubro de 2019, em Foz do Iguaçu-PR. 